\title{
Squamous Alveolar Lining Cell
}

National Cancer Institute

\section{Source}

National Cancer Institute. Squamous Alveolar Lining Cell. NCI Thesaurus. Code C13144.

A flat, scale-like epithelial cell that forms the inner lining of the alveoli. 